\title{
Predictors of hospital mortality in a population-based cohort of patients with acute lung injury*
}

\author{
Colin R. Cooke, MD, MSc; Jeremy M. Kahn, MD, MSc; Ellen Caldwell, MSc; Valdelis N. Okamoto, MD, PhD; \\ Susan R. Heckbert, MD, PhD; Leonard D. Hudson, MD; Gordon D. Rubenfeld, MD, MSc
}

Objective: Studies describing predictors of mortality in patients with acute lung injury were primarily derived from selected academic centers. We sought to determine the predictors of mortality in a population-based cohort of patients with acute lung injury and to characterize the performance of current severity of illness scores in this population.

Design: Secondary analysis of a prospective, multicenter, population-based cohort.

Setting: Twenty-one hospitals in Washington State.

Patients: The cohort included 1,113 patients with acute lung injury identified during the year 1999-2000.

Interventions: None.

Measurements and Main Results: We evaluated physiology, comorbidities, risk factors for acute lung injury, and other variables for their association with death at hospital discharge. Bivariate predictors of death were entered into a multiple logistic regression model. We compared Acute Physiology and Chronic Health Evaluation (APACHE) II, APACHE III, and Simplified Acute Physiology Score II to the multivariable model using area under the receiver operating characteristic curve. The model was vali- dated in an independent cohort of 886 patients with acute lung injury. Modified acute physiology score, age, comorbidities, arterial $\mathrm{pH}$, minute ventilation, $\mathrm{PaCO}_{2}, \mathrm{PaO}_{2} / \mathrm{FIO}_{2}$ ratio, intensive care unit admission source, and intensive care unit days before onset of acute lung injury were independently predictive of in-hospital death $(p<.05)$. The area under the receiver operating characteristic curve for the multivariable model was superior to that of APACHE III (.81 vs. .77, $p<.001)$ but was no different after external validation (.71 vs. $.70, p=.64)$.

Conclusions: The predictors of mortality in patients with acute lung injury are similar to those predictive of mortality in the general intensive care unit population, indicating disease heterogeneity within this cohort. Accordingly, APACHE III predicts mortality in acute lung injury as well as a model using variables selected specifically for patients with acute lung injury. (Crit Care Med 2008; 36:1412-1420)

KEY WORDS: acute respiratory distress syndrome; severity of illness index; risk adjustment; risk factors; prognosis; Acute Physiology and Chronic Health Evaluation; critical illness; epidemiology; regression analysis

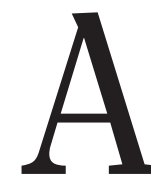

cute lung injury (ALI) is a major cause of respiratory failure associated with significant morbidity and mortality. With a frequency of $18-79$ cases per 100,000 person-years (1-3), ALI is estimated to be responsible for death in up to $75,000 \mathrm{pa}-$ tients per year in the United States, the majority of whom are cared for in nonacademic hospitals (3).
Research on prognostic variables and scoring systems in ALI is important for clinical practice and in the development of research tools (4). Although it is unlikely that clinical decisions in the intensive care unit (ICU) will be driven solely by objective prognostic data, this information is invaluable in informing clinical discussions. Accounting for prognostic variables in clinical trials and adjusting

Foundation \& Ortho Biotech Clinical Affairs, LLC Clinical Research Trainee Award in Critical Care, Northbrook, Illinois.

Dr. Rubenfeld has consulted for and received data from Cerner. The remaining authors have not disclosed any potential conflicts of interest.

Address requests for reprints to: Colin R. Cooke, MD, MSc, Division of Pulmonary \& Critical Care Medicine, Harborview Medical Center, University of Washington, 325 9th Avenue, Box 359762, Seattle, WA 98104. E-mail: crcooke@u.washington.edu

Copyright $(2008$ by the Society of Critical Care Medicine and Lippincott Williams \& Wilkins

DOI: 10.1097/CCM.0b013e318170a375 for their confounding effects in observational studies are central to valid research $(5,6)$.

Investigators confront many choices in selecting approaches to measuring the severity of critical illness (7). There are generic scores, for example, Acute Physiology and Chronic Health Evaluation (APACHE) or Simplified Acute Physiology Score (SAPS), developed to assess heterogeneous populations of critically ill patients. There are also disease-specific scores, for example, the Injury Severity Score or the Model End-Stage Liver Disease Score, developed specifically for traumatic injury and hepatic failure. One can use the scores derived from points assigned to specific variables or use the scores and other variables along with coefficients to develop a predicted risk of death from a regression model. Typically, investigators use the scores rather than a predicted risk of death from a regression model because the scores are easier to calculate, perform well compared with 
more complex models, and may be less subject to secular trends and local practice variation $(4,8)$.

Previous work evaluating risk factors for death in ALI are limited by the inclusion of patients only from referral centers affiliated with academic institutions (915). Studies exploring prognostic variables and severity scores in populationbased cohorts of patients with ALI are limited (1, 2). Recent evidence suggests that ALI patients cared for in nonacademic hospitals are more numerous, are older, have greater severity of illness, and have a different distribution of ALI risk factors than patients cared for in academic ICUs (3). At least one large research collaborative studying ALI has adopted the APACHE III score as a measure of severity of illness; however, data on the performance of this measure in a defined cohort of patients with ALI are lacking (16). No validated, diseasespecific, severity of illness measure for ALI exists, and the need for such a score has not been evaluated.

To address these issues, we used a population-based cohort of patients with ALI to determine the predictors of hospital mortality, to attempt to use this information to develop a customized severity of illness measure for ALI, and to compare generic severity of illness scores with this customized model. We were not interested in the performance of the severity of illness regression models or the predicted risks of death generated by these models.

\section{MATERIALS AND METHODS}

The institutional review board of the University of Washington approved the study.

\section{Study Population}

Patients were drawn from the King County Lung Injury Project (KCLIP) (3). The methods and results of this project were previously reported (3). Briefly, KCLIP is a large, multicenter, prospective cohort study of the frequency and outcome of ALI in King County, WA. All mechanically ventilated patients in 21 hospitals in King County or neighboring counties that care for King County residents were screened for ALI during April 1999 to July 2000 using consensus criteria (17).

\section{Variable Collection and Data Quality}

Pertinent demographic, comorbidity, laboratory, and physiology data were abstracted from the patients' computerized or paper medical record by trained staff using a protocol at the time of enrollment. Comorbidities and operative status were abstracted and coded using the APACHE II or III methodology $(18,19)$. We did not evaluate treatmentrelated variables, such as tidal volume, insulin infusion, tracheostomy, or corticosteroids for their potential causal role in ALI outcome, because these therapies were not strongly supported by evidence at the time of the study and they require a completely different approach to modeling to address confounding and indication bias. ALI risk factors were gathered from the medical record at the time of ALI onset using standard definitions from the 4 days preceding ALI onset (discussed later). Data from day 3 after ALI onset were prospectively abstracted when available. Day 3 differences (day 3 minus onset day) in continuous covariates were calculated when both were measured and were dichotomized as "improved" or "worsened or no difference." Vital status (alive vs. dead) was determined at the time of hospital discharge. Data quality was ensured by standardized training of chart abstractors, double data entry, and random quality checks.

\section{Variable Definitions}

Pulmonary. ALI-related variables evaluated for predictive ability included ventilator variables, arterial blood gases, chest radiography severity, and the timing of ALI onset. Because nearly all patients in this cohort met diagnostic criteria for multiple risk factors during the risk period, risk factors for ALI were modeled using three strategies. First, all ALI risk factors were included in the model as dichotomous covariates. Second, we classified the ALI risk factor as pulmonary/nonpulmonary. Third, we assigned each patient a mutually exclusive, primary ALI risk factor using a prespecified algorithm based on the Injury Severity Score, timing of ALI, sepsis criteria, and presence of other risks. For example, a patient with an Injury Severity Score $>15$ developing ALI within 4 days of injury was assigned a trauma primary risk even if he or she met physiologic criteria for sepsis. Alternate risk factor assignments were explored in sensitivity analyses.

Physiology. We modeled nonpulmonary physiology by modeling all nonrespiratory components of the APACHE III acute physiology score (APS) as ordered-categorical variables. We also modified the APS by subtracting all respiratory points and included it as a linear covariate in the logistic equation, which allowed the pulmonary physiologic variables to take on unique weighting.

Comorbidity. Comorbidities were modeled using three strategies. First, all candidate diseases were entered as dichotomous predictors. Second, the sum-total of the APACHE III chronic health variables was included as a single linear covariate. Third, a Charlson comorbidity score was entered as a continuous covariate (20).

\section{Statistical Analysis}

Bivariate. Variables were evaluated for their association with hospital mortality using chi-square, Student's $t$-test, or Wilcoxon's rank-sum tests as appropriate $(p<.05)$. We calculated APACHE II (18), APACHE III (19), and SAPS II (21) using data from the 24-hr period surrounding ALI onset. This time point replicates when severity scores are commonly calculated in clinical trials. In addition, because most patients developed ALI within 24 hrs of ICU admission, there were no significant differences between severity scores calculated at ICU admission and ALI onset. Each point score was calibrated to the KCLIP data by incorporating it into a separate logistic model as a single covariate in its best fitting form with hospital death as the outcome. SAPS III and the Sequential Organ Failure Assessment scores were not evaluated because they were not calculable in our validation cohort. The APACHE IV score is identical to APACHE III and is therefore not presented (22).

Multivariable. For the regression, we excluded $90(8 \%)$ patients due to missing comorbidities $(\mathrm{n}=19)$, minute ventilation $(\mathrm{n}=$ $69)$, and $\mathrm{PaCO}_{2}(\mathrm{n}=2)$ at the time of ALI onset, leaving 1,023 evaluable patients. Candidate variables associated with death on bivariate analysis $(p \leq .25)$ were included in a multiple logistic regression model (23). Multiple variable modeling used a stepwise, backward elimination, forward entering algorithm retaining significant variables $(p<.05)$. Continuous variables were modeled using fractional polynomials averting the potential bias involved in prespecifying the functional form $(24,25)$. We compared models using the Akaike information criterion and the likelihood ratio chisquare test as appropriate $(23,26)$. No firstorder interaction terms were significant. We calculated predicted probabilities of death by evaluating the regression equation for each individual and plotted predicted mortality against $\mathrm{PaO}_{2} / \mathrm{FIO}_{2}$ ratio. We evaluated model discrimination using the area under the receiver operating characteristic curve (AUC) (27). Comparisons of AUCs used the method of DeLong et al (28). We evaluated model calibration using the Hosmer-Lemeshow chisquare statistic ( $p>.05$ for all models) (23). All tests for significance were two-tailed.

\section{Validation}

We used the bootstrap to internally validate the final model by sampling with replacement for 1,000 iterations (29). The logistic model was fit on each bootstrap sample by repeating the stepwise selection algorithm and then evaluated on the original cohort to estimate the degree to which the predictive accu- 
racy would deteriorate when the final model is applied to an independent sample (29). External validation was accomplished by applying our model to the Acute Respiratory Distress Syndrome Clinical Network's (ARDSNet) low tidal volume study (30).

All analyses were conducted using Stata version 9.2 (Stata, College Station, TX).

\section{RESULTS}

During the KCLIP study period, 1,113 patients met consensus criteria for ALI and were $>15$ yrs old. Four hundred twenty-nine (38.5\%) patients died during the hospitalization in which their ALI was diagnosed. Median (interquartile range) time to hospital death after ALI onset in nonsurvivors was 6 days (2-13 days). Overall mean $(\mathrm{SD})$ tidal volume on day 3 after the onset of ALI for the cohort was $10.2(2.6) \mathrm{mL} / \mathrm{kg}$ predicted body weight and $8.5(2.6) \mathrm{mL} / \mathrm{kg}$ measured body weight, similar to current cohorts described in the literature $(31,32)$. In patients with recorded plateau pressure ( $\mathrm{n}=686), 71 \%$ had values $\leq 30 \mathrm{~cm} \mathrm{H}_{2} \mathrm{O}$ on the day of ALI onset.

\section{Bivariate Analysis}

Patients who were dead at hospital discharge were older than survivors and had greater severity of illness (Table 1). Patients dying had statistically significantly worse respiratory variables, including greater minute ventilation, higher plateau pressure, lower $\mathrm{PaO}_{2} / \mathrm{FIO}_{2}$ ratio, lower arterial $\mathrm{pH}$, and lower arterial $\mathrm{PaCO}_{2}$, at the time of ALI onset. The majority of surviving patients were discharged to skilled nursing or rehabilitation facilities $(51 \%)$, while fewer were discharged to home (34\%) or to longterm acute care facilities or other hospitals $(13 \%)$.

Further details of the mortality and relative risk of death by ALI risk factor, chronic comorbidities, physiology, and hospital and ICU admission source are shown in Table 2. With the exception of sepsis, severe trauma, and other/none, none of the primary ALI risk factors had statistically significant associations with hospital death.

Comorbidities significantly associated with mortality included various malignancies, liver disease, congestive heart failure, and immunosuppression. Other subgroups of patients in the cohort identified at the time of ALI onset with particularly high and low mortality rates are
Table 1. Baseline characteristics of King County Lung Injury Project Cohort by vital status at hospital discharge

\begin{tabular}{|c|c|c|c|}
\hline \multirow[b]{2}{*}{ Variable $^{a}$} & \multicolumn{2}{|c|}{$\begin{array}{c}\text { Vital Status at Hospital } \\
\text { Discharge }\end{array}$} & \multirow[b]{2}{*}{$P$ value } \\
\hline & Dead & Alive & \\
\hline Cases & $\mathrm{n}=429$ & $\mathrm{n}=684$ & \\
\hline Age, years, median (IQR) & $67(52-77)$ & $59(46-73)$ & $<.001$ \\
\hline Male $(\%)$ & 62 & 60 & .6 \\
\hline Race $(\%)$ & & & .1 \\
\hline White & 67 & 70 & \\
\hline Black & 10 & 9 & \\
\hline Asian/Pacific Islander & 9 & 5 & \\
\hline Other/unknown & 15 & 15 & \\
\hline Body mass index & $26.7(7.4)$ & $28.1(7.8)$ & .01 \\
\hline ALI risk factor $(\%)$ & & & $<.001$ \\
\hline Severe sepsis & 79 & 68 & \\
\hline Trauma & 3.5 & 7.3 & \\
\hline None/other & 17 & 25 & \\
\hline \multicolumn{4}{|l|}{ Severity of illness } \\
\hline APACHE III score & $106(31)$ & $76(27)$ & $<.001$ \\
\hline APACHE II score & $30(8)$ & $23(7)$ & $<.001$ \\
\hline SAPS II score & $54(18)$ & $40(16)$ & $<.001$ \\
\hline ICU days pre-ALI, median (IQR) & $1(1-3)$ & $1(1-2)$ & .01 \\
\hline \multicolumn{4}{|l|}{ Respiratory variables (at ALI onset) } \\
\hline Minute ventilation $(\mathrm{L} / \mathrm{min})$ & $12(5)$ & $11(4)$ & $<.001$ \\
\hline Plateau pressure (mm $\mathrm{Hg})$ & $27.1(8.2)$ & $25.5(7.2)$ & .01 \\
\hline $\mathrm{PaO}_{2} / \mathrm{FiO}_{2}$ ratio $(\mathrm{mm} \mathrm{Hg})$ & $140(67)$ & $161(66)$ & $<.001$ \\
\hline $\mathrm{pH}$ & $7.35(.12)$ & $7.39(.10)$ & $<.001$ \\
\hline $\mathrm{PaCO}_{2}(\mathrm{~mm} \mathrm{Hg})$ & $40(11)$ & $42(11)$ & $<.001$ \\
\hline Type of operation (\%) & & & .003 \\
\hline Emergent & 9 & 15 & \\
\hline Non-emergent & 7 & 11 & \\
\hline No operation & 84 & 74 & \\
\hline \multicolumn{4}{|l|}{ Disposition (\%) } \\
\hline Skilled nursing or rehab facility & - & 51 & \\
\hline Home & - & 34 & \\
\hline Hospital or LTAC & - & 13 & \\
\hline Other/unknown & - & 3 & \\
\hline
\end{tabular}

ICU, intensive care unit; IQR, interquartile range; ALI, acute lung injury; APACHE, Acute Physiology and Chronic Health Evaluation; SAPS, Simplified Acute Physiology Score; LTAC, long-term acute care facility.

${ }^{a}$ Data were missing for body mass index in 288 (26\%) patients; severity of illness measures, 19 (0.7\%); plateau pressure, 427 (38\%); $\mathrm{pH}$ and $\mathrm{PaCO}_{2}, 22(2 \%)$; minute ventilation 69 (8\%). ${ }^{b}$ Numbers reflect mean (SD) unless otherwise noted. Percentages may not add to 100 due to rounding.

shown in Table 2 . To contrast the survival experience of ALI patients at relatively low (severe trauma) and high risk (oliguric renal failure) of death compared with the entire cohort, we plotted the time after ALI diagnosis vs. probability of hospital survival in these groups using the Kaplan-Meier estimator (Fig. 1).

\section{Day 3 After ALI Onset}

Of the 1,113 patients in the cohort, $79 \%$ were alive and ventilated on day 3 of ALI. Of these, $100 \%$ had calculable APS, $83 \%$ had recorded minute ventilation, $81 \%$ had available arterial blood gases, and $70 \%$ had recorded positive endexpiratory pressure at both ALI onset and day 3 of ALI. Unadjusted relative risks of death at hospital discharge in patients experiencing an improvement in respiratory/physiology-related variables by day 3 after ALI onset are shown in Table 3.

\section{Multivariable Analysis}

Plateau pressure was not evaluated in the multivariable model due to missing values on day $1(38 \%)$. The prognostic role of body mass index is reported for this cohort elsewhere (33). Table 4 displays the variables independently associated $(p<.05)$ with mortality in the multiple logistic regression model and the magnitude of each variable's contribution to model fit (chi-square). Age and modified APS were highly associated with mortality. Comorbidities remaining sigmetastatic cancer, hepatic failure, leukenificant in the multivariable model were 
Table 2. Mortality rate and relative risk of death for patients with and without specific ALI risk factors, comorbidities, physiology at ALI onset, and each admission source

\begin{tabular}{|c|c|c|c|c|c|}
\hline \multirow[b]{2}{*}{ Variable } & \multirow[b]{2}{*}{$\begin{array}{c}\text { N with } \\
\text { Variable }^{a}\end{array}$} & \multicolumn{2}{|c|}{ Mortality } & \multirow[b]{2}{*}{$\begin{array}{l}\text { Relative Risk } \\
\text { of Death }^{b}\end{array}$} & \multirow[b]{2}{*}{$95 \% \mathrm{CI}$} \\
\hline & & $\%$ & $95 \%$ CI & & \\
\hline Entire cohort & 1113 & 39 & $36-41$ & - & - \\
\hline \multicolumn{6}{|l|}{ ALI risk factor } \\
\hline \multicolumn{6}{|l|}{ Severe sepsis } \\
\hline $\begin{array}{l}\text { No sepsis } \\
\text { Non-pulmonary }\end{array}$ & $\begin{array}{r}312 \\
76\end{array}$ & $\begin{array}{l}29 \\
54\end{array}$ & $\begin{array}{l}24-34 \\
42-65\end{array}$ & $\begin{array}{l}1.00 \\
1.87\end{array}$ & $\begin{array}{l}\text { Sepsis Referen } \\
1.43-2.45\end{array}$ \\
\hline Pulmonary & 416 & 43 & $38-47$ & 1.48 & $1.20-1.81$ \\
\hline Mixed & 309 & 39 & $34-45$ & 1.36 & $1.09-1.70$ \\
\hline Trauma & 65 & 23 & $14-35$ & .58 & $0.37-0.92$ \\
\hline Pancreatitis & 32 & 41 & $24-59$ & 1.06 & $.69-1.62$ \\
\hline Witnessed aspiration & 32 & 38 & $21-56$ & .97 & $.62-1.53$ \\
\hline Pneumonia (non-septic) & 21 & 14 & $3-36$ & .37 & $.13-1.05$ \\
\hline Massive transfusion & 15 & 40 & $16-68$ & 1.03 & $.56-1.94$ \\
\hline Overdose & 14 & 29 & $8-58$ & .74 & $.32-1.69$ \\
\hline Post-cardiac bypass surgery & 10 & 20 & $3-56$ & .52 & $.15-1.79$ \\
\hline Other/none & 123 & 28 & $21-37$ & .71 & $.53-0.96$ \\
\hline \multicolumn{6}{|l|}{ Chest radiograph } \\
\hline AECC definition & 741 & 35 & $32-39$ & 1.00 & Referent \\
\hline $\begin{array}{c}>50 \% \text { alveolar opacity in } 3 \\
\text { or more guadrants }\end{array}$ & 372 & 45 & $40-50$ & 1.27 & $1.09-1.47$ \\
\hline \multicolumn{6}{|l|}{ Comorbidity } \\
\hline Leukemia or lymphoma & 32 & 75 & $57-89$ & 1.99 & $1.61-2.47$ \\
\hline Metastatic cancer & 58 & 69 & $55-80$ & 1.86 & $1.54-2.25$ \\
\hline Chronic renal insufficiency & 42 & 60 & $43-74$ & 1.57 & $1.21-2.03$ \\
\hline Immunosuppressed & 95 & 56 & $45-66$ & 1.50 & $1.23-1.83$ \\
\hline HIV positive & 27 & 56 & $35-75$ & 1.45 & $1.03-2.05$ \\
\hline Liver failure or cirrhosis & 96 & 53 & $43-63$ & 1.42 & $1.16-1.74$ \\
\hline Congestive heart failure & 169 & 47 & $40-55$ & 1.27 & $1.06-1.52$ \\
\hline Non- metastatic cancer & 163 & 45 & $38-53$ & 1.21 & $1.00-1.46$ \\
\hline Diabetes mellitus & 237 & 39 & $33-46$ & 1.02 & $.85-1.22$ \\
\hline \multicolumn{6}{|l|}{ Physiologyc } \\
\hline Highest APS quartile & 273 & 66 & $60-71$ & 2.20 & $1.92-2.52$ \\
\hline Vasopressor use $>2$ hours & 316 & 56 & $51-62$ & 1.78 & $1.55-2.05$ \\
\hline $\mathrm{GCS} \leq 8$ & 402 & 53 & $48-58$ & 1.72 & $1.49-1.99$ \\
\hline Shock & 487 & 50 & $45-54$ & 1.66 & $1.43-1.93$ \\
\hline$\& \mathrm{GCS} \leq 8$ & 183 & 63 & $56-70$ & 1.87 & $1.62-2.16$ \\
\hline$\&$ oliguric renal failure & 65 & 72 & $60-83$ & 1.97 & $1.66-2.34$ \\
\hline Oliguric renal failure & 89 & 69 & $58-78$ & 1.90 & $1.61-2.23$ \\
\hline$\& \mathrm{GCS} \leq 8$ & 48 & 79 & $65-90$ & 2.15 & $1.82-2.53$ \\
\hline$\&$ bilirubin $>2.0 \mathrm{mg} / \mathrm{dL}$ & 15 & 80 & $52-96$ & 2.10 & $1.62-2.74$ \\
\hline Bilirubin $>2.0 \mathrm{mg} / \mathrm{dL}$ & 96 & 64 & $53-73$ & 1.75 & $1.47-2.08$ \\
\hline$\& \mathrm{GCS} \leq 8$ & 43 & 74 & $59-86$ & 2.00 & $1.65-2.42$ \\
\hline Arterial $\mathrm{pH}<7.20$ & 76 & 66 & $54-76$ & 1.80 & $1.50-2.15$ \\
\hline Minute ventilation $>20 \mathrm{~L} / \mathrm{min}$ & 43 & 65 & 49-79 & 1.71 & $1.36-2.16$ \\
\hline $\mathrm{PaO}_{2} / \mathrm{FIO}_{2} \leq 100 \mathrm{~mm} \mathrm{Hg}$ & 307 & 50 & $44-56$ & 1.46 & $1.26-1.69$ \\
\hline \& shock & 164 & 58 & $50-66$ & 1.64 & $1.40-1.92$ \\
\hline$\&$ oliguric renal failure & 28 & 71 & $51-87$ & 1.89 & $1.48-2.42$ \\
\hline \multirow{2}{*}{\multicolumn{6}{|c|}{ Hospital admission source (\%) }} \\
\hline & & & & & \\
\hline Home & 838 & 39 & $36-42$ & 1.00 & Referent \\
\hline Other hospital & 95 & 33 & $23-42$ & .84 & $.62-1.13$ \\
\hline Skilled nursing/other facility & 161 & 42 & $34-49$ & 1.07 & $.87-1.31$ \\
\hline \multicolumn{6}{|l|}{ ICU admission source } \\
\hline Emergency room & 496 & 35 & $31-39$ & 1.00 & Referent \\
\hline Ward & 272 & 52 & $46-58$ & 1.48 & $1.25-1.74$ \\
\hline Direct & 62 & 47 & $34-60$ & 1.33 & $1.00-1.78$ \\
\hline Operating room/angiography & 253 & 30 & $24-36$ & .86 & $.69-1.07$ \\
\hline Other & 11 & 36 & $11-70$ & 1.04 & $.47-2.29$ \\
\hline \multicolumn{6}{|l|}{ Other variable combinations } \\
\hline Any malignancy & 242 & 54 & $47-60$ & 1.56 & $1.34-1.81$ \\
\hline Age $<45$ and trauma as ALI risk & 37 & 16 & $6-32$ & .41 & $.20-.86$ \\
\hline Age $\geq 65$ years & 499 & 46 & $41-50$ & 1.39 & $1.20-1.62$ \\
\hline$\& \mathrm{PaO}_{2} / \mathrm{FIO}_{2} \leq 100 \mathrm{~mm} \mathrm{Hg}$ & 119 & 64 & $55-72$ & 1.80 & $1.53-2.10$ \\
\hline$\&$ highest APS quartile & 114 & 69 & $60-78$ & 1.97 & $1.70-2.28$ \\
\hline Age $\geq 80$ years & 153 & 51 & $43-59$ & 1.39 & $1.17-1.66$ \\
\hline$\& \mathrm{PaO}_{2} / \mathrm{FIO}_{2} \leq 100 \mathrm{~mm} \mathrm{Hg}$ & 30 & 77 & $58-90$ & 2.04 & $1.65-2.52$ \\
\hline \& highest APS quartile & 33 & 73 & $54-87$ & 1.93 & $1.54-2.51$ \\
\hline
\end{tabular}

ALI, acute lung injury; AECC, American European Consensus Conference; CI, confidence interval; HIV, human immunodeficiency virus; APS, APACHE III acute physiology score; GCS, Glasgow coma score.

${ }^{a}$ Results available for 1094 patients except for: minute ventilation $(\mathrm{n}=1025)$; age, $\mathrm{PaO}_{2}: \mathrm{FiO}_{2}$, ALI risk factor, chest radiograph $(\mathrm{n}=1113) ; \mathrm{pH}, \mathrm{PaCO}_{2}(\mathrm{n}=1091) .{ }^{b}$ Relative risk comparing $\mathrm{N}$ with variable to all other patients unless otherwise noted. ${ }^{c}$ Shock defined as mean arterial pressure $\leq 60$ $\mathrm{mm} \mathrm{Hg}$ at any point during onset day. Oliguric renal failure defined as the combination of urine output $\leq 500 \mathrm{cc}$ in a 24 -hr period and serum creatinine $\geq 2.0 \mathrm{mg} / \mathrm{dl}$ during onset day. mia, lymphoma, and congestive heart failure. Patients admitted from the ward to the ICU had an odds ratio of $1.55(95 \%$ confidence interval 1.06-2.28) compared with those admitted from the emergency room. Patients developing ALI after 48 hrs in the ICU had an $82 \%$ greater odds of death (odds ratio 1.82, 95\% confidence interval 1.32-2.50) compared with those developing ALI within the first $48 \mathrm{hrs}$ independent of other factors. The $\mathrm{PaO}_{2} /$ $\mathrm{FIO}_{2}$ ratio was best modeled using a fractional polynomial. The independent odds and predicted probability of death increased dramatically below $\mathrm{PaO}_{2} / \mathrm{FIO}_{2}$ $<100 \mathrm{~mm} \mathrm{Hg}$ (Fig. 2). Chest radiography severity and ALI risk factor, regardless of coding method, were not associated with mortality after adjustment for other factors, including admission source and respiratory variables. The Hosmer-Lemeshow goodness-of-fit statistic for this customized model showed no evidence of lack of fit $\left(\chi_{8}^{2}=11.15, p=.19\right)$.

The AUCs for the customized model and other severity of illness measures are shown in Table 5. The customized model (Table 4) had statistically better mortality discrimination compared with APACHE II, APACHE III, and SAPS II. The AUC for APACHE III was superior to APACHE II $(p=.004)$ and SAPS II $(p=.001)$. After internal validation using the bootstrap, the AUC for the customized model dropped to 0.79 (95\% confidence interval $.76-.81)$. External validation through application of the customized model to the ARDSNet low tidal volume study $(\mathrm{n}=$ 886) resulted in an AUC of .71 (.67-.74) compared with .70 (.67-.74) for APACHE III. This difference did not reach statistical significance $(p=.64)$. Calibration of the customized model to the ARDSNet cohort showed adequate goodness of fit $\left(\chi^{2}{ }_{8}=8.53, p=.38\right)$ as well as for APACHE III $\left(\chi^{2}{ }_{8}=9.45, p=.31\right)$.

\section{DISCUSSION}

In this population-based cohort of patients with ALI, the primary determinants of hospital mortality were similar to those seen in other populations of critically ill patients. Acute physiologic derangement, age, severe chronic comorbidities, ICU admission source, and duration of ICU stay before ALI onset were all important independent predictors of mortality. Certain subgroups, including patients with coma, liver dysfunction, renal dysfunction, shock, and profound hypoxemia, have particularly 




Figure 1. Kaplan-Meier estimates of hospital survival among 65 patients with severe trauma and 89 patients with oliguric renal failure (defined as urine output $<500 \mathrm{~mL}$ in a 24 -hr period and serum creatinine $>2.0 \mathrm{mg} / \mathrm{dL}$ ) at onset of acute lung injury ( $A L I)$ compared with 1,113 patients in the entire cohort. Curves are not mutually exclusive. Patients were censored at hospital discharge. Time is truncated at 120 days.

Table 3. Relative risk (RR) of death at hospital discharge in patients experiencing improvement in respiratory/physiologic variables by day three post-acute lung injury onset

\begin{tabular}{|c|c|c|c|c|c|}
\hline \multirow{2}{*}{$\begin{array}{l}\text { Variable (day three compared } \\
\text { to onset day) }{ }^{a}\end{array}$} & \multirow{2}{*}{$\begin{array}{l}\mathrm{n} \text { in Category/ } \\
\text { Total N }\end{array}$} & \multicolumn{2}{|c|}{ Mortality } & \multicolumn{2}{|c|}{ RR of Death } \\
\hline & & $\%$ & $95 \% \mathrm{CI}$ & Point & $95 \% \mathrm{CI}$ \\
\hline \multicolumn{6}{|l|}{ APS } \\
\hline Lower & $581 / 876$ & 33 & $29-37$ & .77 & $.64-.91$ \\
\hline Greater or no difference & $295 / 876$ & 43 & $38-49$ & 1.00 & Referent \\
\hline \multicolumn{6}{|l|}{$\mathrm{PaO}_{2} / \mathrm{FIO}_{2}$} \\
\hline Greater & $482 / 710$ & 32 & $28-36$ & .74 & $.61-.90$ \\
\hline Lower or no difference & $228 / 710$ & 43 & $35-50$ & 1.00 & Referent \\
\hline \multicolumn{6}{|l|}{ Minute ventilation } \\
\hline Lower & $338 / 730$ & 36 & $31-42$ & .89 & $.74-1.07$ \\
\hline Greater or no difference & $392 / 730$ & 41 & $36-46$ & 1.00 & Referent \\
\hline \multicolumn{6}{|l|}{ PEEP } \\
\hline Lower & $85 / 615$ & 32 & $22-43$ & .79 & $.57-1.10$ \\
\hline Greater or no difference & $530 / 615$ & 40 & $36-45$ & 1.00 & Referent \\
\hline \multicolumn{6}{|l|}{$\mathrm{PaCO}_{2} \mathrm{P}-\mathrm{P}$} \\
\hline Greater & $300 / 709$ & 33 & $27-38$ & .88 & $.72-1.08$ \\
\hline Lower or no difference & $409 / 709$ & 37 & $32-42$ & 1.00 & Referent \\
\hline
\end{tabular}

APS, Acute Physiology and Chronic Health Evaluation III acute physiology score; CI, confidence interval; PEEP, positive end-expiratory pressure.

${ }^{a}$ Each variable represents the day three value minus the day one value dichotomized at zero. "Lower" denotes negative difference. "Greater" denotes positive difference. Patients with no difference are grouped in the referent category for each variable.

poor prognosis; however, no risk factors either alone or in combination sufficiently identified patients whose care was futile. Among patients who survived to and were intubated on day 3 , those with improving physiology were less likely to die. In external validation, a regression model using the generic APACHE III score recalibrated to this cohort performed as well as a customized model that incorporated ALI-specific variables, including risk factor for ALI and pulmonary physiology.

Consistently identified independent predictors of mortality in previous stud- ies of ALI include age $(9,34)$, nonpulmonary organ dysfunction $(12,14)$, and liver disease $(9,34)$. Additional predictors of mortality, such as body mass index (33, 35), pulmonary deadspace fraction (13), immunosuppression (14), ALI risk factor $(10,11,15)$, and ventilator days before ALI (9), have also been identified, yet many were not isolated as independent predictors in subsequent studies. Previous studies examining predictors of mortality evaluated patients from tertiary care academic centers with few focusing on population-based cohorts $(1,34)$. We determined that nonpulmonary organ dysfunction; age; history of leukemia, congestive heart failure, or hepatic failure; arterial pH; ICU admission source; ICU stay prior to ALI onset $>48 \mathrm{hrs}$; minute ventilation; and $\mathrm{PaCO}_{2}$ are all independently predictive of mortality. These results are important because they demonstrate that in a population-based cohort of patients with ALI, predictors of mortality are similar to large heterogeneous cohorts of patients with critical illness from around the world.

Contrary to some other studies, we did not find that ALI risk factor was an independent predictor of death $(10,11,15)$. This was true regardless of the method that we used to code risk factor (primary risk, any risk, or pulmonary/nonpulmonary risk). Although ALI risk factor was associated with death on bivariate analyses, it dropped out of the multivariable analysis. Other variables in the model, including acute physiology, oxygenation, ICU admission source, and comorbidity, likely capture the information provided by the risk factor for ALI. This finding agrees with the only other populationbased study to rigorously evaluate the association between ALI risk factor and death (34).

We determined that the $\mathrm{PaO}_{2} / \mathrm{FiO}_{2}$ ratio at ALI onset was independently associated with mortality. This finding disagrees with most $(13,14,34,36)$ but not all $(1,37)$ previous studies in ALI. The discrepancy between our results and those of others may result from the power of this large ALI cohort to detect such an effect, our richer $\mathrm{PaO}_{2} / \mathrm{FIO}_{2}$ modeling strategy using fractional polynomials $(25,38,39)$, our use of a modified APS to prevent collinearity between physiology modeling and oxygenation (13, 14, $34)$, and underlying differences between KCLIP and cohorts in previous studies. In the analysis by Luhr and colleagues (1) of 221 patients with acute respiratory dis- 
Table 4. Multivariate model with adjusted odds ratios (OR) and $95 \%$ confidence intervals (CI) for independent predictors of mortality in King County Lung Injury Project

\begin{tabular}{|c|c|c|c|c|}
\hline Variable & & Adjusted OR & $95 \% \mathrm{CI}$ & Covariate $\chi^{2 a}$ \\
\hline Modified acute physiology score (per point) & & 1.03 & $1.02-1.04$ & 100.11 \\
\hline Age (per 10 years older) & & 1.25 & $1.14-1.37$ & 23.31 \\
\hline Metastatic tumor & & 4.94 & $2.51-9.75$ & 23.07 \\
\hline Hepatic failure & & 3.06 & $1.46-6.40$ & 9.03 \\
\hline Lymphoma & & 4.22 & $1.21-14.71$ & 5.29 \\
\hline Leukemia & & 9.82 & $1.87-51.70$ & 9.63 \\
\hline Congestive heart failure & & 1.77 & $1.17-2.66$ & 7.47 \\
\hline Admission source to ICU & & & & 7.05 \\
\hline Emergency room & & 1.00 & Referent & \\
\hline Ward & & 1.55 & $1.06-2.28$ & \\
\hline Direct & & 1.36 & $.73-2.55$ & \\
\hline OR/angiography & & .93 & $.62-1.38$ & \\
\hline Other & & 1.03 & $.22-4.73$ & \\
\hline Time in ICU prior to ALI onset (hours) & & & & 14.01 \\
\hline$\leq 48$ & & 1.00 & Referent & \\
\hline$>48$ & & 1.82 & $1.32-2.50$ & \\
\hline Arterial pH (per 0.1 more alkalotic) & & .81 & $.68-.96$ & 5.82 \\
\hline $\mathrm{PaO}_{2} / \mathrm{FiO}_{2}$ ratio $^{a, b}$ & Reference point & & & 17.41 \\
\hline $1-100$ & 50 & 3.04 & $1.74-5.31$ & \\
\hline $101-150$ & 125 & 1.15 & $1.07-1.23$ & \\
\hline $151-200$ & 175 & 1.05 & $1.02-1.08$ & \\
\hline $201-300$ & 250 & 1.00 & Referent & \\
\hline $\mathrm{PaCO}_{2}$ (per 5-mm Hg increase) & & .90 & $.83-.99$ & 5.2 \\
\hline Minute ventilation $(\mathrm{L} / \mathrm{min})$ & & & & 8.22 \\
\hline$<9$ & & .58 & $.40-.85$ & \\
\hline $9-12$ & & 1.00 & Referent & \\
\hline$>12$ & & .87 & $.59-1.28$ & \\
\hline
\end{tabular}

ALI, acute lung injury; ICU, intensive care unit; OR, operating room.

${ }^{a}$ Likelihood ratio $\chi^{2}$ statistic reflects relative contribution of the covariate to the overall model fit. ${ }^{b}$ Modeled as a function of $\mathrm{P} / \mathrm{F}$ ratio $\left.\left((\mathrm{P} / \mathrm{F} 100)^{\wedge}-2\right)-0.426\right)$ as a continuous covariate using fractional polynomials. The odds ratio $(\mathrm{OR})$ for each category was calculated by comparing the midpoint of the given range (reference point) to the midpoint of the last category (P/F 250).

tress syndrome identified through a population-based screening of patients in Scandinavia, those with a $\mathrm{PaO}_{2} / \mathrm{FIO}_{2}$ ratio $>100 \mathrm{~mm}$ Hg at enrollment had an odds of death $26 \%$ lower than those with $\mathrm{PaO}_{2} /$ $\mathrm{FIO}_{2}<100 \mathrm{~mm} \mathrm{Hg}$. This finding, like ours, was independent of age, ALI risk factor, and physiologic perturbation. In contrast to a subsequent analysis by Luhr and colleagues (34), we found that patients whose oxygenation improved by day 3 of ALI were at lower odds of death.

Our results also support the hypothesis that hypercapnia may be protective or that hypocapnia is injurious in ALI. Each 5-mm Hg increase in the $\mathrm{PCO}_{2}$ was associated with a $10 \%$ reduction in the odds of hospital death independent of $\mathrm{pH}$, minute ventilation, and other factors. Although these results corroborate prior work (40), our a priori analysis plan did not seek to exhaustively evaluate this association; thus, we did not control for plateau pressure or other variables that might specifically confound this association.

Our results illustrate the considerable overlap between the predictors of death in ALI and predictors of death in general ICU populations throughout the world. Additional significant variables in our multivariable model, including arterial $\mathrm{pH}$, leukemia, admission source to the ICU, and duration of stay before ALI onset, are not well-described predictors of death in ALI patients. These variables, however, are well-known predictors of death in the general ICU population (22, 41). In fact, with the exception of minute ventilation, all of the predictors of mortality in our multivariable model were previously described in at least one of the three largest current ICU severity of illness measures $(19,22,41)$.

There are several reasons why the predictors of death in ALI are similar to those in the general ICU population. First, ALI is a syndrome resulting from a heterogeneous group of insults. Typical ALI cohorts include not only young healthy patients with trauma but also elderly patients with pneumonia or sepsis $(30,42)$. The APACHE III prognostic equation was derived on a heterogeneous group of $>17,000$ critically ill patients that included $>70$ ICU admission diag- noses (19), and SAPS III was derived using $>16,000$ patients in 303 ICUs across five continents. Both of these severity measures incorporated ALI patients during their derivation. In their populationbased study of respiratory failure, Luhr and colleagues (34) determined that nonpostoperative mechanical ventilation, in the absence of ALI, is associated with a mortality of $41 \%$, which is similar to our reported mortality of $38.5 \%$ in ALI. Moreover, experts' ability to clinically separate ALI from other heterogeneous causes of respiratory failure is limited $(34,43)$. Given the heterogeneous nature of ALI and acute respiratory failure, it is understandable that the predictors of mortality given by these severity scores would overlap with those in ALI.

Second, patients who develop acute lung injury do not often die of hypoxic respiratory failure but rather die as a result of their underlying disease or, more important, as a result of withdrawal from mechanical ventilation (44). As we described here, the majority of variables in our multivariable model simply reflect an ICU patient's severity of illness. Therefore, our inability to isolate ALI-specific clinical predictors of death in this large cohort may reflect the fact that in a broad population base, none exist.

Third, our results show that APACHE II and SAPS II do not discriminate between patients who will live and those who will die as well as the APACHE III score or our customized model. This result has important implications to the scientific community. Although ARDSNet has adopted APACHE III as the preferred severity of illness measure gathered during its studies, the use of APACHE II and SAPS II in ALI research remains common $(35,45,46)$. The importance of the small improvement in discrimination seen with the APACHE III score over APACHE II or SAPS II is unknown. However, since the APACHE III score is openly available and easily calculable, investigators planning studies of ALI should consider using it over other severity of illness measures. Risk adjusting with alternative scores may not fully capture a patient's severity of illness and could lead to residual confounding.

There are a number of limitations to our analysis. First, our patient population was gathered during the year 1999-2000, before publication of a landmark study showing that low tidal volume ventilation reduced mortality in ALI (30). Predictors of mortality in ALI patients ventilated 

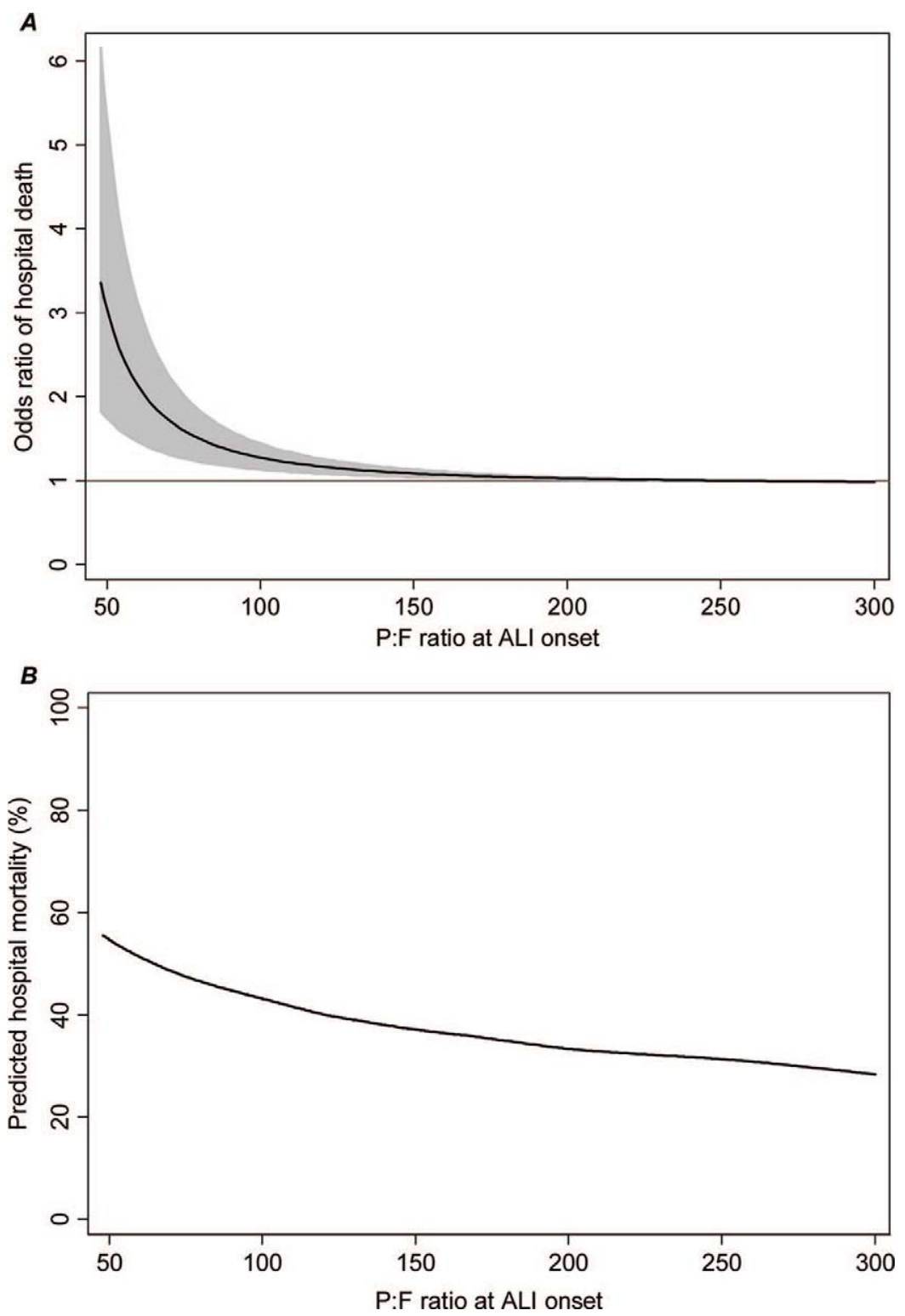

Figure 2. Relationship between $\mathrm{PaO}_{2} / \mathrm{FIO}_{2}(P: F)$ ratio at onset of acute lung injury $(A L I)$ and riskadjusted odds and probability of hospital death. Risk adjustment variables included in the model are modified acute physiology score, age, comorbidities (leukemia, lymphoma, metastatic cancer, hepatic failure, congestive heart failure), duration of intensive care unit stay before ALI, location before intensive care unit admission, arterial $\mathrm{pH}$, minute ventilation, and $\mathrm{PaCO}_{2} . A$, the solid line indicates the point estimate for the odds ratio for each P:F on the $\mathrm{x}$-axis. The gray region indicates the $95 \%$ confidence interval for the point estimate. The risk-adjusted odds of death are presented for each P:F relative to the midpoint of the reference category (P:F 201-300 mm Hg). Area above the horizontal line indicates increased hospital mortality relative to patients with P:F of $250 \mathrm{~mm} \mathrm{Hg}$ and below the horizontal line indicates lower hospital mortality. $B$, the line represents a smoothed estimate of the predicted probability of hospital death for the cohort. Smoothing achieved using locally weighted least squares (lowess).

with this strategy may differ than those in our study. However, recent studies continue to suggest that there is limited uptake of low tidal volume ventilation in patients with ALI, and tidal volumes in this cohort mimic those seen in other more recent cohorts of patients with ALI, suggesting generalizability of our findings $(31,32)$.
Table 5. Comparison of area under the receiver operating characteristic curve (ROC) for customized model and other severity of illness measures prior to validation

\begin{tabular}{lcc}
\hline & \multicolumn{2}{c}{$\begin{array}{c}\text { Area Under the ROC } \\
\text { Curve }\end{array}$} \\
\cline { 2 - 3 } \multicolumn{1}{c}{ Score $^{a}$} & Original & $95 \%$ CI \\
\hline Customized model $^{b}$ & .81 & $.78-.83$ \\
APACHE III & $.77^{c}$ & $.75-.80$ \\
APACHE II & $.75^{c}$ & $.72-.78$ \\
SAPS II & $.74^{c}$ & $.71-.77$ \\
\hline
\end{tabular}

${ }^{a}$ Scores calculated from the 24 -hr period surrounding day of ALI onset. ${ }^{b}$ Refers to the model shown in Table $4 .{ }^{c} p<0.001$ compared to customized model.

ALI, acute lung injury; APACHE, Acute Physiology and Chronic Health Evaluation; CI, confidence interval; SAPS, Simplified Acute Physiology Score.

and various biological markers (6). Our customized model was only able to improve upon APACHE III to the extent that the additional comorbidities and respiratory-specific variables gathered during the study contribute to prediction of death. In addition to examining generic ICU severity of illness scoring, future research on prognostic modeling in patients with ALI should explore novel genetic and physiologic markers and other biomarkers and pay specific attention to how these evolve over time.

Third, because we used patients arising from a population base to determine the predictors of mortality in ALI, our ability to determine academic and community-specific predictors of death was limited. Academic and community hospitals care for different patient populations, and different patient populations generate different risk equations. Nevertheless, we believe that our predictors generalize to the entire spectrum of patients with ALI.

Fourth, we were unable to evaluate some potentially important predictors because they were not collected routinely in this cohort. Plateau pressure, a complex variable linked to physician management decisions as well as underlying physiology, was not incorporated into the model because it was missing in 38\% of the cohort on day 1 . We were also unable to evaluate recently developed severity of illness scores, such as SAPS III, which may outperform both APACHE III and our model. 


\section{CONCLUSIONS}

We found that there is considerable overlap between the predictors of mortality in patients with ALI and predictors in general ICU patients. In addition, APACHE III is an appropriate risk adjustment tool for patients with ALI. Future attempts to develop a severity of illness measure in ALI should incorporate novel clinical, biological, or genetic data.

\section{ACKNOWLEDGMENTS}

The Acute Respiratory Distress Syndrome Clinical Network (ARDSNet) is conducted and supported by the National Heart, Lung, and Blood Institute (NHLBI) in collaboration with the ARDSNet study investigators. This report was prepared in part using a limited-access dataset obtained from the NHLBI and does not necessarily reflect the opinions or views of the ARDSNet study or the NHLBI.

\section{REFERENCES}

1. Luhr OR, Antonsen K, Karlsson M, et al: Incidence and mortality after acute respiratory failure and acute respiratory distress syndrome in Sweden, Denmark, and Iceland. The ARF Study Group. Am J Respir Crit Care Med 1999; 159:1849-1861

2. Bersten $\mathrm{AD}$, Edibam $\mathrm{C}$, Hunt $\mathrm{T}$, et al: Incidence and mortality of acute lung injury and the acute respiratory distress syndrome in three Australian states. Am J Respir Crit Care Med 2002; 165:443-448

3. Rubenfeld GD, Caldwell E, Peabody E, et al: Incidence and outcomes of acute lung injury. N Engl J Med 2005; 353:1685-1693

4. Herridge MS: Prognostication and intensive care unit outcome: The evolving role of scoring systems. Clin Chest Med 2003; 24: 751-762

5. Pocock SJ, Assmann SE, Enos LE, et al: Subgroup analysis, covariate adjustment and baseline comparisons in clinical trial reporting: Current practice and problems. Stat Med 2002; 21:2917-2930

6. Ware LB: Prognostic determinants of acute respiratory distress syndrome in adults: Impact on clinical trial design. Crit Care Med 2005; 33:S217-S222

7. Rubenfeld GD: Severity of illness measures. In: Clinical Critical Care Medicine. Albert RK, Slutsky AS, Ranieri VM, et al (Eds). Philadelphia, Mosby-Elsevier, 2006, pp 95-100

8. Wunsch H, Linde-Zwirble WT, Angus DC: Methods to adjust for bias and confounding in critical care health services research involving observational data. J Crit Care 2006; $21: 1-7$

9. Monchi M, Bellenfant F, Cariou A, et al: Early predictive factors of survival in the acute respiratory distress syndrome: A multivariate analysis. Am J Respir Crit Care Med 1998; 158:1076-1081

10. Vieillard-Baron A, Girou E, Valente E, et al: Predictors of mortality in acute respiratory distress syndrome: Focus on the role of right heart catheterization. Am J Respir Crit Care Med 2000; 161:1597-1601

11. Eisner MD, Thompson T, Hudson LD, et al: Efficacy of low tidal volume ventilation in patients with different clinical risk factors for acute lung injury and the acute respiratory distress syndrome. Am J Respir Crit Care Med 2001; 164:231-236

12. Rocco TR Jr, Reinert SE, Cioffi W, et al: A 9 -year, single-institution, retrospective review of death rate and prognostic factors in adult respiratory distress syndrome. Ann Surg 2001; 233:414-422

13. Nuckton TJ, Alonso JA, Kallet RH, et al: Pulmonary dead-space fraction as a risk factor for death in the acute respiratory distress syndrome. N Engl J Med 2002; 346:1281-1286

14. Brun-Buisson C, Minelli C, Bertolini G, et al: Epidemiology and outcome of acute lung injury in European intensive care units: Results from the ALIVE study. Intensive Care Med 2004; 30:51-61

15. Moran JL, Solomon PJ, Fox V, et al: Modelling thirty-day mortality in the acute respiratory distress syndrome (ARDS) in an adult ICU. $A n$ aesth Intensive Care 2004; 32:317-329

16. Knaus WA, Sun X, Hakim RB, et al: Evaluation of definitions for adult respiratory distress syndrome. Am J Respir Crit Care Med 1994; 150:311-317

17. Bernard GR, Artigas A, Brigham KL, et al: The American-European Consensus Conference on ARDS: Definitions, mechanisms, relevant outcomes, and clinical trial coordination. Am J Respir Crit Care Med 1994; 149:818-824

18. Knaus WA, Draper EA, Wagner DP, et al: APACHE II: A severity of disease classification system. Crit Care Med 1985; 13:818-829

19. Knaus WA, Wagner DP, Draper EA, et al: The APACHE III prognostic system: Risk prediction of hospital mortality for critically ill hospitalized adults. Chest 1991; 100:1619-1636

20. Charlson ME, Pompei P, Ales KL, et al: A new method of classifying prognostic comorbidity in longitudinal studies: Development and validation. J Chronic Dis 1987; 40:373-383

21. Le Gall JR, Lemeshow S, Saulnier F: A new Simplified Acute Physiology Score (SAPS II) based on a European/North American multicenter study. JAMA 1993; 270: 2957-2963

22. Zimmerman JE, Kramer AA, McNair DS, et al: Acute Physiology and Chronic Health Evaluation (APACHE) IV: Hospital mortality assessment for today's critically ill patients. Crit Care Med 2006; 34:1297-1310

23. Hosmer DW, Lemeshow S: Applied Logistic Regression. New York, Wiley, 2000

24. Royston P, Sauerbrei W: A new approach to modelling interactions between treatment and continuous covariates in clinical trials by using fractional polynomials. Stat Med 2004; 23:2509-2525

25. Royston P, Sauerbrei W: Building multivariable regression models with continuous covariates in clinical epidemiology - with an emphasis on fractional polynomials. Methods Inf Med 2005; 44:561-571

26. Akaike H: Information theory and an extension of the maximum likelihood principle. In: Petrov BN, Csaki F (Eds). 2nd International Symposium on Information Theory, Budapest: Akademiai Kiado, 1973: 267-281

27. Zweig MH, Campbell G: Receiver-operating characteristic (ROC) plots: A fundamental evaluation tool in clinical medicine. Clin Chem 1993; 39:561-577

28. DeLong ER, DeLong DM, Clarke-Pearson DL: Comparing the areas under two or more correlated receiver operating characteristic curves: A nonparametric approach. Biometrics 1988; 44:837-845

29. Steyerberg EW, Harrell FE Jr, Borsboom GJ, et al: Internal validation of predictive models: Efficiency of some procedures for logistic regression analysis. J Clin Epidemiol 2001; 54:774-781

30. Ventilation with lower tidal volumes as compared with traditional tidal volumes for acute lung injury and the acute respiratory distress syndrome. The Acute Respiratory Distress Syndrome Network. N Engl J Med 2000; 342: 1301-1308

31. Sakr Y, Vincent JL, Reinhart K, et al: High tidal volume and positive fluid balance are associated with worse outcome in acute lung injury. Chest 2005; 128:3098-3108

32. Yilmaz M, Keegan MT, Iscimen R, et al: Toward the prevention of acute lung injury: Protocol-guided limitation of large tidal volume ventilation and inappropriate transfusion. Crit Care Med 2007; 35:1660-1666

33. Morris AE, Stapleton RD, Rubenfeld GD, et al: The association between body mass index and clinical outcomes in acute lung injury. Chest 2007; 131:342-348

34. Luhr OR, Karlsson M, Thorsteinsson A, et al: The impact of respiratory variables on mortality in non-ARDS and ARDS patients requiring mechanical ventilation. Intensive Care Med 2000; 26:508-517

35. O'Brien JM Jr, Phillips GS, Ali NA, et al: Body mass index is independently associated with hospital mortality in mechanically ventilated adults with acute lung injury. Crit Care Med 2006; 34:738-744

36. Krafft P, Fridrich P, Pernerstorfer T, et al: The acute respiratory distress syndrome: Definitions, severity and clinical outcome: An analysis of 101 clinical investigations. Intensive Care Med 1996; 22:519-529

37. Spragg RG, Lewis JF, Walmrath HD, et al: Effect of recombinant surfactant protein Cbased surfactant on the acute respiratory distress syndrome. $N$ Engl $J$ Med 2004; 351: 884-892

38. Royston P, Altman DG, Sauerbrei W: Dichotomizing continuous predictors in multiple 
regression: A bad idea. Stat Med 2006; 25 : 127-141

39. Royston P, Ambler G, Sauerbrei W: The use of fractional polynomials to model continuous risk variables in epidemiology. Int J Epidemiol 1999; 28:964-974

40. Kregenow DA, Rubenfeld GD, Hudson LD, et al: Hypercapnic acidosis and mortality in acute lung injury. Crit Care Med 2006; 34:1-7

41. Moreno RP, Metnitz PG, Almeida E, et al: SAPS 3-From evaluation of the patient to evaluation of the intensive care unit: Part 2.
Development of a prognostic model for hospital mortality at ICU admission. Intensive Care Med 2005; 31:1345-1355

42. Gattinoni L, Caironi P, Cressoni M, et al: Lung recruitment in patients with the acute respiratory distress syndrome. $N$ Engl $\mathrm{J} \mathrm{Med}$ 2006; 354:1775-1786

43. Rubenfeld GD, Caldwell E, Granton J, et al: Interobserver variability in applying a radiographic definition for ARDS. Chest 1999; 116:1347-1353

44. Stapleton RD, Wang BM, Hudson LD, et al:
Causes and timing of death in patients with ARDS. Chest 2005; 128:525-532

45. Kacmarek RM, Wiedemann HP, Lavin PT, et al: Partial liquid ventilation in adult patients with acute respiratory distress syndrome. Am J Respir Crit Care Med 2006; 173:882-889

46. Perkins GD, McAuley DF, Thickett DR, et al: The beta-agonist lung injury trial (BALTI): A randomized placebo-controlled clinical trial. Am J Respir Crit Care Med 2006; 173: 281-287 